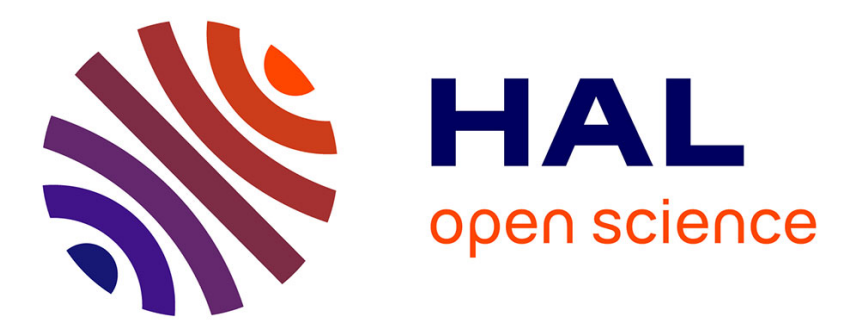

\title{
High Q 2D-length extension mode resonators for potential time-frequency applications
}

Paul Chapellier, Pierre Lavenus, Olivier Le Traon, Bernard Dulmet

\section{To cite this version:}

Paul Chapellier, Pierre Lavenus, Olivier Le Traon, Bernard Dulmet. High Q 2D-length extension mode resonators for potential time-frequency applications. Microsystem Technologies, 2019, pp.1-12. 10.1007/s00542-019-04575-0 . hal-02470334

\section{HAL Id: hal-02470334 \\ https://hal.science/hal-02470334}

Submitted on 7 Feb 2020

HAL is a multi-disciplinary open access archive for the deposit and dissemination of scientific research documents, whether they are published or not. The documents may come from teaching and research institutions in France or abroad, or from public or private research centers.
L'archive ouverte pluridisciplinaire HAL, est destinée au dépôt et à la diffusion de documents scientifiques de niveau recherche, publiés ou non, émanant des établissements d'enseignement et de recherche français ou étrangers, des laboratoires publics ou privés. 


\title{
High Q 2D-Length Extension Mode Resonators for potential Time-Frequency applications
}

\section{Paul Chapellier, Pierre Lavenus, Olivier Le Traon}

ONERA the French Aerospace Lab, Physics, Space, Environment, Instrumentation Department, Sensors and Microtechnology unit

29, avenue de la Division 92320 Châtillon, France

\section{Bernard Dulmet}

FEMTO-ST Institute, UMR CNRS 6174, Time and Frequency Department

15B, avenue des Montboucons, 25000 Besançon, France

paul.chapellier@onera.fr +33(0) 146734730

\begin{abstract}
This paper presents recent advances on two dimensional Length-Extension Mode (2D-LEM) quartz resonators providing high quality $(Q)$ factor on resonances at a few MHz. The resonators have been collectively manufactured using one or two steps quartz Deep Reactive-Ion Etching (DRIE) processes. These resonators combine the intrinsic qualities of quartz in comparison to silicon (i.e. high $Q$ factor, low temperature sensitivity and piezoelectricity) and the advantages of Microelectromechanical systems (MEMS) resonators: small dimensions, low power consumption and collective processes. Samples vibrating at frequencies $f$ of $2.2,3$ and $4.5 \mathrm{MHz}$ have shown promising results with very high $Q$ factor. $Q$ factor as high as 180000 for fundamental mode vibrating at $2.2 \mathrm{MHz}$ and 89000 for harmonic mode at $8.9 \mathrm{MHz}$ were measured which lead to quality factor and resonance frequency products $(Q . f)$ figure of merit near $10^{12} \mathrm{~Hz}$ at the state of the art for 2D-LEM quartz resonators and the higher $Q$ factor measured for DRIE made quartz resonators. Two designs, several dimensions and two processes have been investigated. Two main limiting damping mechanisms were identified and one of them is strongly linked to the technological limits of the etching process.
\end{abstract}

Acknowledgment

This work is partially supported by the Centre National d'Etudes Spatiales CNES. 


\section{INTRODUCTION}

An oscillator is a self-sutaining system capable of generating a signal with a stable frequency. Oscillators are used as frequency references, frequency synthetiser or clocks in almost every electronic devices. They are mandatory in a wide range of fields:watchs, consumer electronics, network equipment, automotive, or embedded systems for space and military applications (Vig2016) An oscillator is composed at least of a frequency selective element, usually an electromechanical resonator that acts like a filter and a gaining element that maintains oscillations.

A resonator is primaly characterized by its resonance frequency in $\mathrm{Hz}$, its quality $(Q)$ factor that reflects the sharpness of the resonance and its serie resistance $\mathrm{R}_{\mathrm{m}}$ in $\Omega$. For oscillator applications, high $Q$ factor is mandatory to ensure low phase noise close to the carrier and low $\mathrm{R}_{\mathrm{m}}$ is important to ensure the start of the oscillations and low phase noise far from the carrier. Other parameters such as frequency stability with temperature, with time (short term or ageing) or pressure are also important to ensure a stable output frequency. The multibillion dollar market of oscillators is dominated by quartz based oscillators that benefit from significant advantages of quartz such as low internal viscous losses (resulting in high $Q$ factor), linear conversion between mechanical and electrical energy through piezoelectricityand high stability versus temperature for well-chosen crystal cuts.

However quartz resonators can be quite bulky and their integration is off-chip leading to a cumbersome packaging and they are somewhat incompatible with emerging applications such as mobile, new telecommunication networks or Internet of Things (IoT) where small size, low cost, low power consumption and medium to high performances are required (Nguyen2007). To overcome these issues silicon $(\mathrm{Si})$ or Thin-film piezoelectric-on-silicon (TPoS) Micro-Electro-Mechanical Sytems resonators have been developed since the end of the 90' (Jokic2015) Altought Si MEMS resonators can show very high $Q$ factor up to several millions at few $\mathrm{MHz}$ (Xereas2015), thermal frequency stability of silicon is pretty poor (around $30 \mathrm{ppm} /{ }^{\circ} \mathrm{C}$ ) and has to be compensated using additionnal material layer or doping. Capacitive transduction leads to high serie resistance (at best few $\mathrm{k} \Omega$ versus 50 to $100 \Omega$ for standard quartz resonators) and induced non-linearities lead to a degradation of the phase noise close from the carrier (Kaajakari2005). TPoS MEMS resonators using AlN or $\mathrm{ZnO}$ on Si bulk show low $Q$ factors,lower than 10000 usually (vanBeek2012). and thermal stability of the stack also needs compensation. On the other hand $\mathrm{R}_{\mathrm{m}}$ is low (from tens of $\Omega$ up to 1 $\mathrm{k} \Omega$ ) thanks to piezoelectricity. The approach for the Si and TPoS MEMS is to use established MEMS processes on well known but not the most suited materials for oscillator applications.

The approach developed in this paper is different as the aim is to use quartz-one of the best if not the best material for oscillator application- with collective processes still under development to manufacture higly miniaturized quartz resonators in order to integrate them in high stability, low cost and power consumption oscillators. As the dimensions are shrinking the standard wet chemical etching using Hydrofluoric acid (HF) and Ammonium Fluoride $\left(\mathrm{NH}_{4} \mathrm{~F}\right)$ is not suitable because of etching induced faceting (Pelle 1998) nor is the chemical-mechanical polishing. This paper deals with the designs of new planar quartz resonators for oscillator applications based on a previous high $Q$ factor bulky resonator originally designed to reach the quantum ground state. The challenging collective processes flow using quartz Deep Reactive Ion Etching (DRIE), the characterizations of the resonators and the comparison with the expected performances are furthermore discussed.

\section{PReVious DESIGN AND MOTIVATIONS}

Reaching the quantum ground state (QGS) with a macroscopic device thus creating a bridge between quatum and classic theories is a topic that many teams and laboratories have been interested in within the last two decades. Devices used are typically nano-micromechanical resonators such as nanotube resonator (Khosla2019), aluminum micromechanical disk resonator (Pirkkalainen2015), silica toroidal resonators (Rivière2010) or aluminu nitride microresonator (Connell2010). Advanced cooling techniques are required to reach such low energy level. Extremely high sensitivity are mandatory to observe quantum fluctuations as well as very high resonators quality factors to ensure long coherence time and long state life time. In a previous work of our group (Le Traon et al 2011), a three dimensional Length-Extension Mode (3D-LEM) quartz resonator was designed, fabricated and characterized to reach the ground state. Figure 1a) shows a cross-section of the 3D-LEM concept for a cylindrical structure: a pillar is vibrating in a length-extensional motion whereas a ring around this pillar is vibrating in an out-of-phase motion allows to compensate the lateral displacement induced by Poisson effect in tethers. This compensating effect leads to reduced displacements and energy losses at the anchors areas. Figure 1b) shows a realization of nine 3D-LEM resonators on a 1.5-inches square Z-cut quartz wafer using wet chemical etching and Figure 1c) shows a detail of the micro-pillar. a triangular pillar was selected to reduce as much as possible the faceting induced by wet chemical etching.

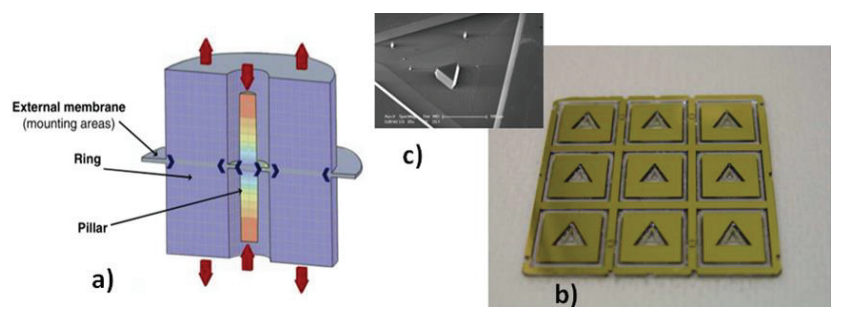

Fig.1 a) Concept of the 3D-LEM: a ring around the pillar with an out-of-phase length-extension vibration, b) 1.5-inches square quartz wafer including nine 3DLEM made using wet etching, c) detail of the micro-pillar. 
—This 3D-LEM quartz resonator exhibited a very high $Q$ factor of 2000000 at $4 \mathrm{MHz}$ reaching a Q.f product of $8 \times 10^{12} \mathrm{~Hz}$ near the theoritical limit of quartz of $3.2 \times 10^{13} \mathrm{~Hz}$ in the Akhiezer regime where the predominant losses mechanisms is phonon-phonon interaction (Akhiezer 1939). Thanks to its very high $Q$ factor, this resonator might find a relevant application in another field such as Time and Frequency where very high $Q$ factor quartz resonator are usually used to control oscillators for timing and frequency references. The best resonators used in Ultra Stable Oscillators (USO) are usually bulky $\left(>200 \mathrm{~mm}^{3}\right) \mathrm{shear}^{\mathrm{mode}} \mathrm{Stress}$ Compensated (SC) or Temperature Compensated (AT) cut quartz resonators with very high $Q$ up to $2.6 \times 10^{6}$ at $5 \mathrm{MHz}(\mathrm{Besson}$ $1995)$ to ensure high frequency stability. The 3D-LEM resonator performances are close to that and that is why this resonator could be a potential candidate for Time and Frequency applications. Unfortunately, the 3D-LEM resonator is also quite bulky ( $>100$ $\mathrm{mm}^{3}$ ) and its 3D design does not allow electrical actuation. Compact planar two dimensional length-extension mode (2D-LEM) resonators using similar working principle have been designed to overcome this issues and should be suited for the realization of quartz micro-resonator based MEMS oscillators (Bourgeteau2014).

\section{NEW RESONATORS DESIGNS AND QUALITY FACTOR}

\subsection{Mode of interest}

2D-LEM resonators of type I and II consist of a beam vibrating in extension-compression. The resonance frequency is around a few $\mathrm{MHz}$ with a central beam of few millimeters. More precisely, the resonance of the fundamental mode $(\mathrm{n}=0)$ and harmonics for Z-cut quartz are given by the well-known equation at the first order (1):

$$
f_{2 n+1}=\frac{2 n+1}{L} \sqrt{\frac{1}{s_{22} \rho}}=(2 n+1) \frac{2.72}{L} M H z
$$

where $L$ is the total length of the vibrating beam in mm, $n$ is the mode number, $\rho$ the quartz density and $s_{22}$ the elastic compliance along Y-axis. As seen from (1), the resonance frequency can be adjusted depending on the targeted applications by varying $L$. This should be done though homothetic transformations of the whole structure to maintain the optimized decoupling between the beam and the mounting area. For the ease of processing, resonators are actuated via gold electrodes only deposited on the upper surface. Thanks to the quartz piezoelectric coupling an electric field applied along X-axis induces a strain along Y-axis as it can be seen in Figure 2 .

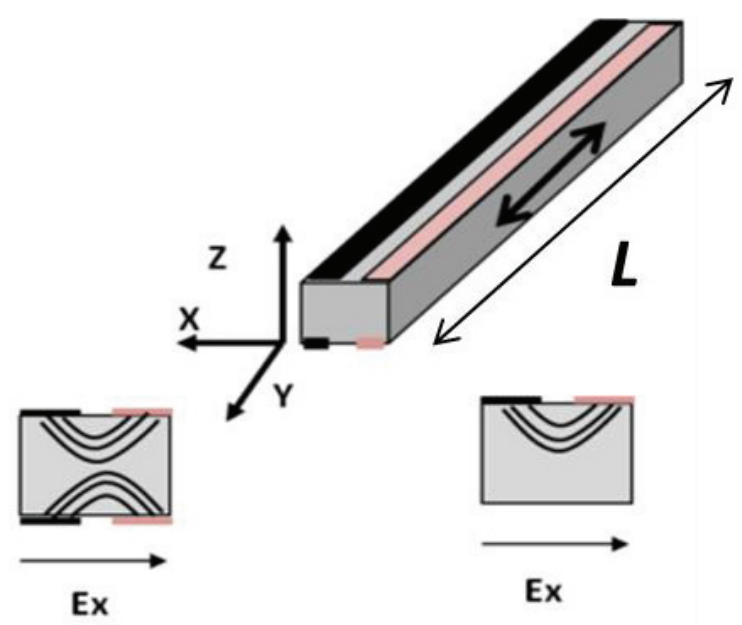

Quartz Z-cut

Fig.2 Electrode scheme for the actuation of the extension mode of a Quartz Z-cut beam oriented along the Y-axis.

\subsection{Resonators designs}

\subsubsection{First design, "type I" resonator}

In addition to the central beam, the type I resonator has two large and short lateral beams that vibrate in an in-plane forced flexure movement that compensates for the shear stress induced through Poisson effect by the vibrating central beam. Figure 3a shows the amplified nodal displacements of type I and II resonators using the Finite Element Method (FEM) simulations with the Multiphysics software OOFELIE and the graphical interface SAMCEF. Thus, the confinement of the stress close to the central beam allows to minimize the vibrating energy in the mounting area and reduces the energy loss towards the mounting which is absolutely necessary to reach the expected high intrinsic quality factor of the quartz resonator. Figure $3 \mathrm{~b}$ shows the X component of the magnified deformation of the beam in a flexure motion and Figure $3 \mathrm{c}$ shows the $\mathrm{Y}$ component of the deformation. Typical nominal dimensions and targeted frequencies of the type I resonator are shown in table 1. 


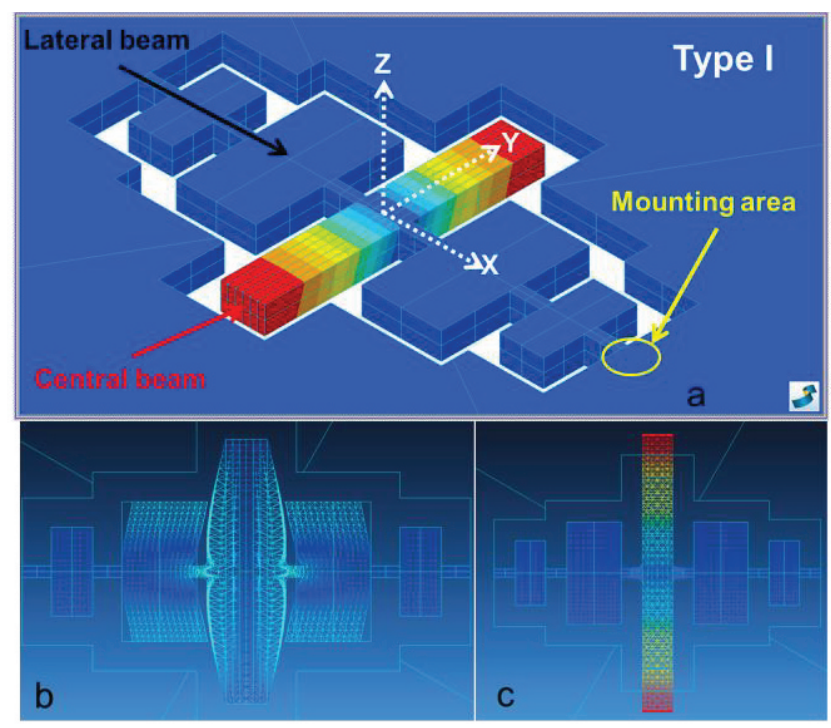

Fig.3 a) Amplified nodal displacements of the fundamental mode of the central beam of a type I resonator during its length-extension mode vibration b) X component of the type I resonator showing the flexure motion of the two lateral beams, c) Y component of the type I resonator showing the extensioncompression displacement of the central beam.

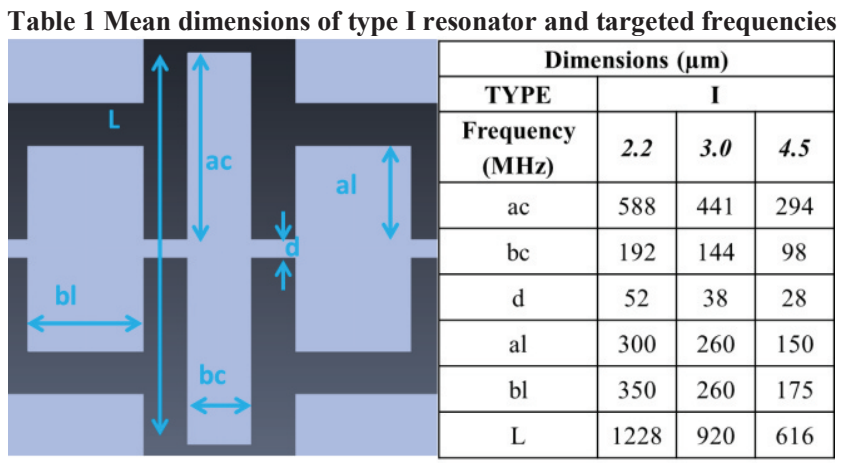

\subsubsection{Second design, "type II” resonator}

In addition to the central beam, the type II resonator has two thin and long lateral beams as shown at the top of Figure 4 . The decoupling between the central beam and the mounting area is partly ensured by a flexure movement similar to the type I resonator. The type II 2D-LEM resonator also benefits from an additional compensation mechanism relying on a length-extension vibration of the lateral beams in opposite phase with respect to the central beam (Le Traon 2013). This is illustrated in Figure 4b and Figure 4c which show respectively the magnified deformations along $\mathrm{X}$ and $\mathrm{Y}$-axis of the type II 2D-LEM resonator. A drawback of this additional decoupling structure is the addition of unwanted eigen modes close to the natural resonance of the central beam corresponding to the eigen modes of the lateral beams which would result in mode coupling if the quality factor is not high enough. Typical nominal dimensions and targeted frequencies of the type II resonator are shown in table 2. 


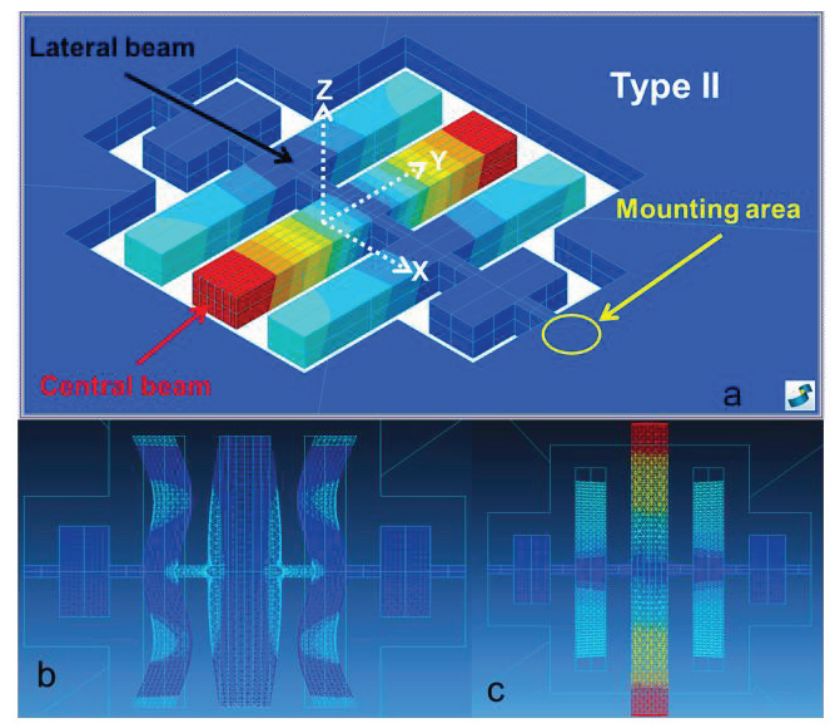

Fig.4 a) Amplified nodal displacements of the fundamental mode of the central beam of a type II resonator during its length-extension mode vibration b) X component of the type II resonator showing the flexure motion of the two lateral beams, d) Y component of the type II resonator showing the extensioncompression displacement of the central beam and the predominant out-of-phase length extension of lateral beams.

Table 2 Mean dimensions of type II resonator and targeted frequencies

\begin{tabular}{ll}
$*$ \\
\multirow{2}{*}{ al }
\end{tabular}

\subsection{Theoritical $Q$ factor}

The $Q$ factor of a resonator can be defined as the ratio of energy stored within the structure over the energy dissipated per cycle via all the different damping mechanisms. Assuming no coupling between the damping mechanisms, energy losses can be written as the sum of the loss induced by the mechanism considered separately, leading to a quality factor that can be written under vacuum as:

$$
\frac{1}{Q}=\frac{1}{Q_{v i s c}}+\frac{1}{Q_{A K E}}+\frac{1}{Q_{T E D}}+\frac{1}{Q_{\text {anchor }}}
$$

where damping mechanisms are briefly described further. A more complete description can be found in (Brand et al, 2015) and an application in the case of silicon beams is provifed in (Le Foulgoc.et al. 2006). Qanchor corresponds to the strain energy lost at the anchors in the mounting areas and is estimated using FEM simulations. By design $Q_{\text {anchor }}$ is very high $\left(>10^{8}\right)$ for a flawless structure. $Q_{T E D}$ is estimated using FEM simulations (Oofelie Solver) able to take into account the thermoelasticity damping. This loss mechanism is due to the bending of the beams which induces a thermal gradient and a strain gradient leading to thermal transport and damping. In the case of 2D-LEM, the flexure motion is not predominant and $Q_{T E D}$ is very high $\left(>10^{7}\right)$. Akhiezer loss $Q_{A K E}$ (Akhiezer 1939) also known as the quantum limit corresponds to the energy loss by phonon-phonon interaction needed to return in a thermodynamics equilibrium due to the lattice deformation induced by the displacement. Q Q AKE is estimated via an analytic expression (Ghaffari et al. 2013) and the $Q$.f product depends on the material. For quartz $Q . f$ reaches $3.2 \times 10^{13} \mathrm{~Hz}$ leading to a $Q_{A K E}$ around $10^{7}$ for structures vibrating at few MHz. Finally, energy loss arises from the viscosity of the gold electrodes which acts as a damping layer. The related $Q_{v i s c}$ can be estimated using an analytical model developed in (Le Traon et al. 2010) and (Bourgeteau et al. 2014) is expected to be the main damping contribution in the 2D-LEM case. With a perfect geometry, a quality factor around 500000 is expected for resonators vibrating at a few MHz making possible to reach a Q.f product between 1.1 and $1.5 \times 10^{12} \mathrm{~Hz}$. 


\section{PROCESS FLOW}

Standard wet etching using Hydrofluoric acid (HF) and Ammonium Fluoride $\left(\mathrm{NH}_{4} \mathrm{~F}\right)$ is not suitable for etching the vibrating part due to its high anisotropy and faceting (Pelle 1998). Deep Reactive Ion Etching of quartz has been developed for the last decade thanks to the development of high density plasma etcher. DRIE-made resonators have been reported for various applications such as Quartz Crystal Microbalance (Kutsuwada et al. 2017) (Abe \& Esashi 2000), gyroscope (Grousset et al. 2014) or timing applications (Kubena et al. 2017) (Kamijo et al. 2014). Vertical etching of quartz over several tens microns is still challenging.

\subsection{Process 1: Two DRIE steps process}

Process 1 is described in Figure 5. Chromium/Gold electrodes are evaporated and patterned, then a bilayer of resins (LOR 10B from MICROCHEM and S1813 from MICROPOSIT) is coated and a $3 \mu \mathrm{m}$ thick nickel mask is sputtered. Lift-off is performed by soaking the samples into hot remover solution (Remover PG from MICROCHEM), once rinsed using isopropanol (IPA) and deionized water samples are ready to be etched. This process uses two DRIE steps: once the patterns of the resonators are made with the first etching step, the wafer is flipped and cavities are etched until the desired thickness and full liberation of the structures. Residual nickel is removed afterwards with nickel etchant solution (TFB from TRANSENE). This DRIE process uses a combination of a fluorocarbon gas tetrafluoromethane $\left(\mathrm{CF}_{4}\right)$ and dioxygen: vertical etching of quartz takes place thanks to the presence of a passivating polymer (Sankaran \& Kushner 2004) (HyunHo 1996) on the sidewalls. Because of the highly directional ion-milling, the passivating layer is barely absent at the bottom of the trenches.

This process suffers from a major issue: the wafer is cracking due to the thermal expansion of nickel submitted to high temperatures during the DRIE etching. This phenomenon has been observed in other work (Boy et al. 2011). The need for strong on-milling and the low thermal conductivity of quartz lead to this excessive heating up to $250^{\circ} \mathrm{C}$ on the quartz wafer.

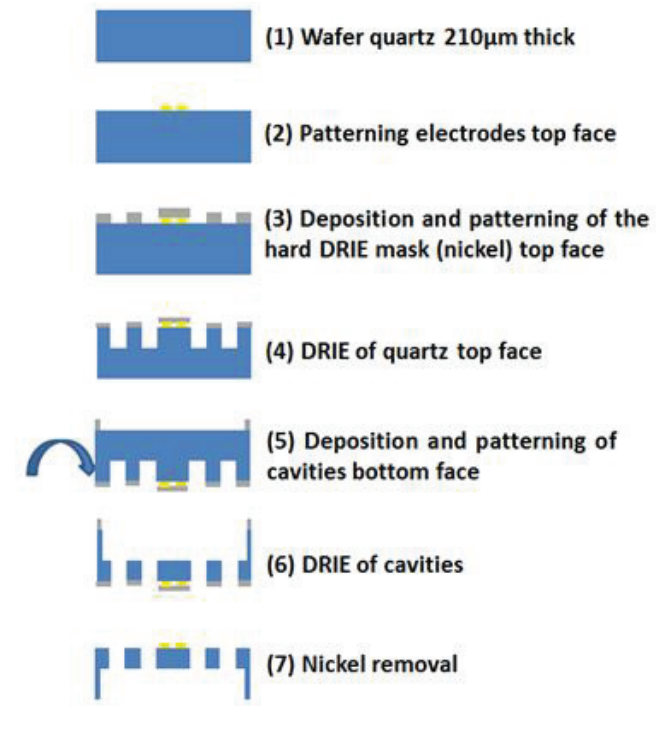

Fig.5 Process flow 1 using two DRIE steps

\subsection{Process 2: One DRIE step process with cavities etched with wet chemical solution}

To reduce the cracking, a process using cavities etched with a wet mixture of Hydrofluoric and Ammonium Fluoride (HF-NH4) has been developed and presented in Figure 6. It allows a reduction of the duration of the DRIE step by at least one half. Chromium/Gold layers are evaporated on both faces as they serve as electrodes and etching mask during the wet chemical etching step. Cavities are patterned and etched using the $\mathrm{HF}-\mathrm{NH}_{4} \mathrm{~F}$ mixture. Then, as in process 1 , a bilayer of resist is coated, nickel is sputtered, lift-off is performed and resonators are etched using single-step DRIE. Only the back side of the vibrating part is left rough as the release of the structure is made by the DRIE step. 

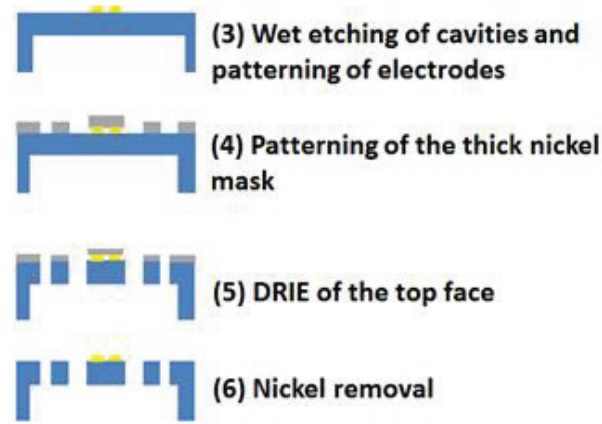

Fig.6 Process flow 2 using wet etching to create cavities and one DRIE step on top face. DRIE parameters are the same as in process 1

\section{RESULTS}

2D-LEM resonators with 3 targeted frequencies have been manufactured using both processes. Figure 7 shows type I resonators on wafer after nickel removal and a focus on a $3.0 \mathrm{MHz}$ resonator. Electrical measurements were performed on the wafer using gold wires bonding under vacuum $\left(10^{-2} \mathrm{~Pa}\right)$ with impedance analyzer (Keysight EA990A). To estimate resonators performances, $Q$ factor, resonance frequencies and serie resistances $\mathrm{R}_{\mathrm{m}}$ are obtained using the standard Butterworth-Van Dyke (BVD) model and are discussed in this section.

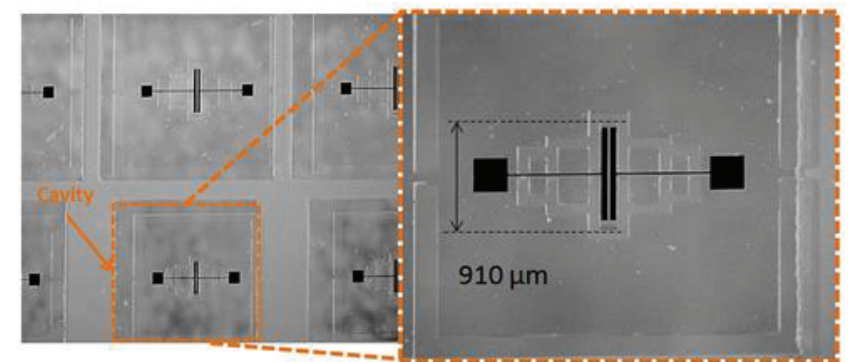

Fig.7 Left, top line type I resonators vibrating at $2.2 \mathrm{MHz}$, bottom line at $3 \mathrm{MHz}$. Right, focus on a $3 \mathrm{MHz}$ resonator. Resonators were manufactured using process 1 .

\subsection{Resonance frequency and measured dimensions}

Mean measured values and standard deviations of resonance frequencies versus the inverse of the total measured central beam length are shown in Figure 8. Data points are aligned with the curve obtained using FEM simulations, meaning that the $f$ - $L$ product is constant and the mode of interest is correctly selected. Lateral dimensions were measured with optical microscopy and the resolution is better than $1 \mu \mathrm{m}$ for the used magnitudes.

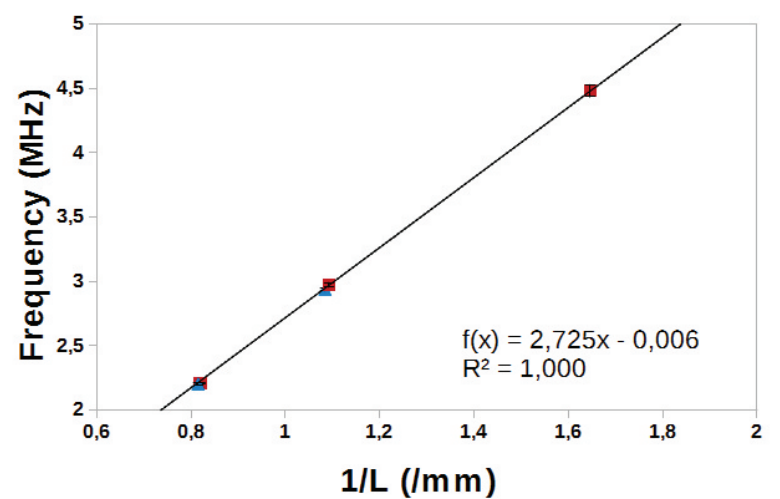

Fig.8 Mean experimental resonance frequencies versus the inverse of the mean experimental total length of the central beam for process 1 (blue triangles), for process 2 (red suares) and simulated frequency versus the inverse of the total length beam of the simulated structure using FEM (dashed black line). 
Experimental resonance frequencies standard deviations were quite similar for both processes and larger for smaller devices $5 \%$ at 4.5 MHz, less than $2 \%$ at $3.0 \mathrm{MHz}$ and less than $1 \%$ for the larger resonators vibrating at $2.2 \mathrm{MHz}$. The trend curve of the simulated structures exhibits a coefficient of $2.725 \mathrm{MHz} . \mathrm{mm}$ for the fundamental mode and is in good agreement with the estimation of equation (1). Figures 9 a), b), c) and d) show optical microscopy images focusing on type I and type II resonators with measured dimensions for 3 and $2.2 \mathrm{MHz}$ resonators obtained with process 1 . In average the measured dimensions of the central and lateral beams are few microns larger than the ones on the lithography mask, this lateral resolution loss is likely due to the resist layers stack and the thick nickel mask. Also the corners of the beams are not straight but slightly rounded. These process-induced flaws are more significant for the smaller resonators at higher frequencies as the resonators dimensions shrink. Some micro-masking is present on the backside cavities corresponding to the dark dots.

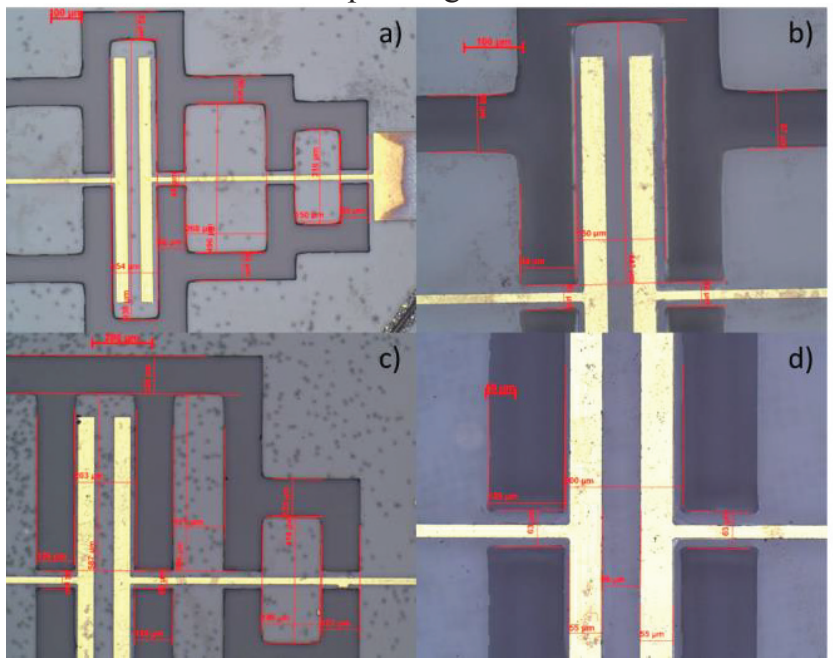

Fig.9 a) Optical image of a type I resonator vibrating at $3 \mathrm{MHz}$ magnification factor $(\mathrm{MF})=100$, b) focus on the central beam MF $=200$, c) Optical image of a type II resonator vibrating at 2.2 MHz MF $=100$, d) focus on the central beam MF $=200$. Resonators were manufactured using process 1 .

Figures 10 a), b), c), d) ,e) and f) show optical microscopy images focusing on type I and type II resonators with measured dimensions for 4.5, 3 and $2.2 \mathrm{MHz}$ resonators obtained with process 2. Black dots on the top face correspond to some residual attack from the wet chemical echting of the cavities and the darker areas correspond to the pyramids on the backside cavities. 


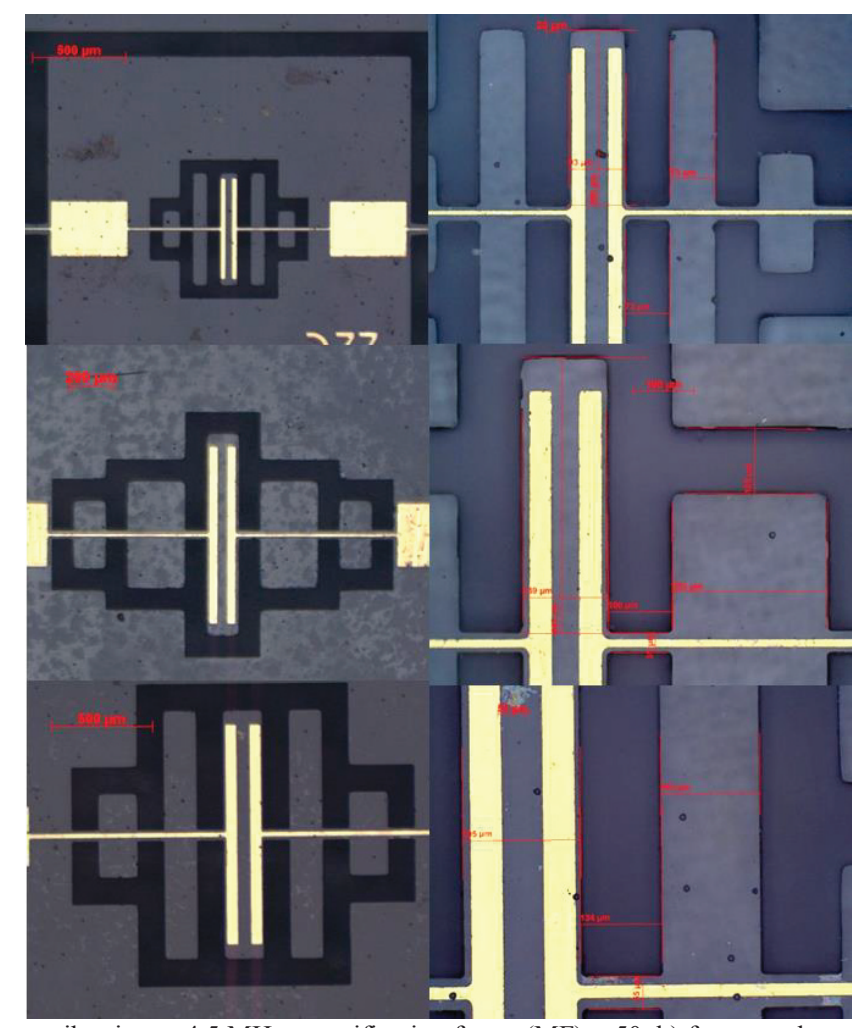

Fig.10 a) Optical image of a type II resonator vibrating at $4.5 \mathrm{MHz}$ magnification factor (MF) $=50$, b) focus on the central beam $\mathrm{MF}=200$, c) Optical image of a type I resonator vibrating at $3 \mathrm{MHz} M F=50$, d) focus on the central beam $\mathrm{MF}=200$, e) Optical image of a type II resonator vibrating at $2.2 \mathrm{MHz}$ magnification factor $(\mathrm{MF})=50, \mathrm{f})$ focus on the central beam $\mathrm{MF}=200$. Resonators were manufactured using process 2 .

\subsection{Experimental Q-factor}

Experimental $Q$ factor were obtained using standard BVD model to fit the data. Figure 11 shows the mean experimental values of $Q$ factors versus the experimental mean resonance frequencies for both types and both processes.

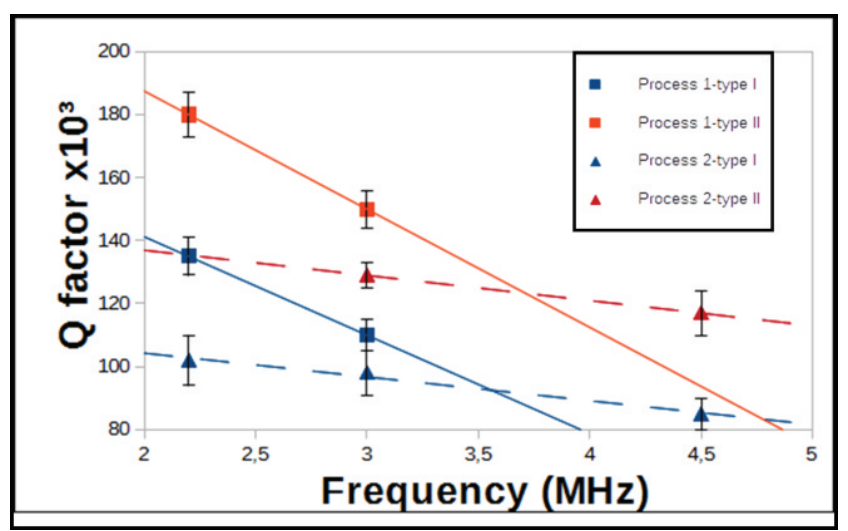

Fig.11 Mean experimental values of $Q$ factors versus mean experimental resonance frequencies for both types and both processes.

\subsubsection{Results of process 1}

Resonators vibrating at 2.2 and $3.0 \mathrm{MHz}$ have been manufactured using process 1 . As expected from (1), the f-L product is a constant at the first order for the different sizes of manufactured resonators. Experimental quality factors as high as 180000 $(\mathrm{Rm}=5.4 \mathrm{k} \Omega)$ for type II resonators vibrating at $2.2 \mathrm{MHz}$ and as high $150000(\mathrm{Rm}=7.5 \mathrm{k} \Omega)$ for type II vibrating at $3.0 \mathrm{MHz}$ were measured. This is however less than half of the expected Q meaning that there is at least another source of damping not included or underestimated in the previous estimation.

\subsubsection{Results of process 2}

The cracking with the process 2 was reduced which allowed the fabrication of smaller resonators - vibrating at $4.5 \mathrm{MHz}-$ and type II resonators vibrating at $4.5 \mathrm{MHz}$ showed best $Q . f$ so far for fundamental mode reaching $5.3 \times 10^{11} \mathrm{~Hz}$ 
$\left(Q=117000, \mathrm{R}_{\mathrm{m}}=9.8 \mathrm{k} \Omega\right)$ and improving the state of the art of 2D-LEM quartz resonators. Yet, resonators showed overall lower quality factors as $Q$ did not exceed $130000\left(\mathrm{R}_{\mathrm{m}}=8.5 \mathrm{k} \Omega\right)$ at $3 \mathrm{MHz}$ and still type II resonators showed better performances.

Triple existe, mais the trip

\subsection{Use of harmonics}

Using harmonics $(n=1,2,3 \ldots$ in Eq (1)) to increase the Q.f product seems an adequate alternative. The first harmonic vibrating at three times the frequency of the fundamental is shown in Figure 12 for type I and II resonators. The central beam is divided into three smaller beams which is equivalent to a central beam of length three times smaller, hence the vibration at thrice the fundamental frequency. The decoupling mechanisms are still the same as in the case of the fundamental mode. Nevertheless, it is important to note that the design of the resonator and in particular the electrode network was optimized to excite the fundamental mode and not the harmonics. On one hand, the decoupling is less efficient, as can be seen in the Figure 8 , lateral beams of the type I resonator and external beams of type II resonator move slightly, which implies an energy loss in the mounting areas. One the other hand, the electrodes array is not optimized for harmonics as a result a low charge collection and high associated motional resistances.

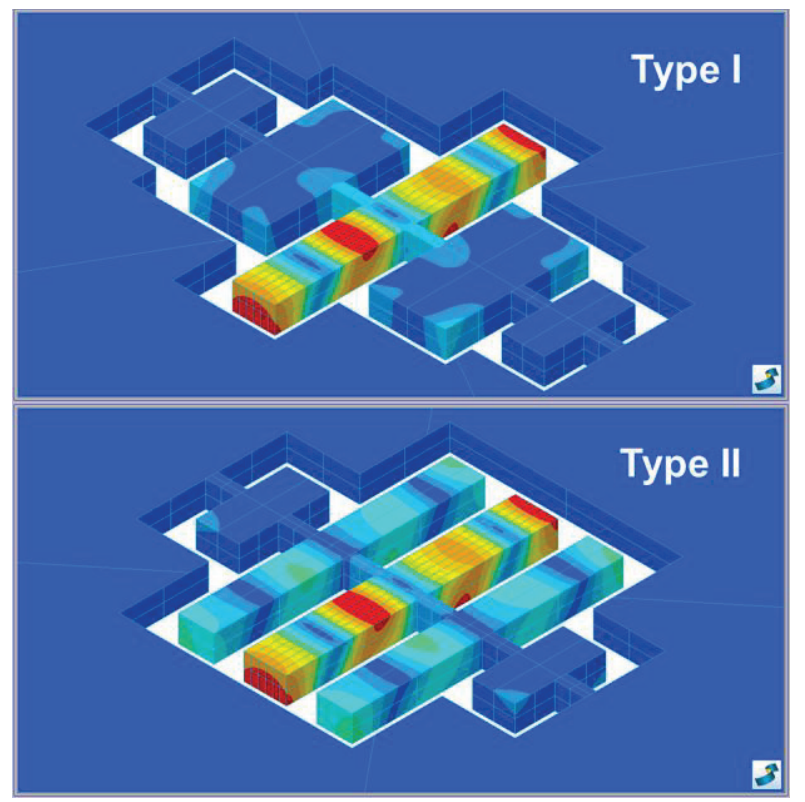

Fig.12 Amplified nodal displacements of the vibrating part obtained using FEM simulations of the first harmonic mode ( $\mathrm{n}=1$ ). Left: type I resonator, right type II resonator

Quality factors as high as $89000\left(\mathrm{R}_{\mathrm{m}}=42.7 \mathrm{k} \Omega\right)$ at $8.8 \mathrm{MHz}$ (fundamental mode vibrating at $2.9 \mathrm{MHz}$ ) and 40000 $(\mathrm{Rm}=101 \mathrm{k} \Omega$ ) at $6.6 \mathrm{MHz}$ (fundamental mode vibrating at $2.2 \mathrm{MHz}$ ) have been measured. The maximum $Q . f$ product reached is $7.8 \times 10^{11} \mathrm{~Hz}$ which is an increase of $50 \%$ compared to the $Q . f$ products obtained with fundamental mode. All the results are presented in the graph of the Figure 13. An optimized design and electrode network are necessary to achieve the full potential of harmonics: increasing the resonance frequency without excessively degrading the quality factor. In fact, as the frequency increases the strain energy stored in the vibrating part is increased whereas the energy contained in the gold layers varies slowly with frequency resulting in higher $Q_{\text {visc }}$. The dependency of the $Q_{\text {anchor }}$ for harmonic modes with the sidewall angle and resonator dimensions will be studied in future work. 


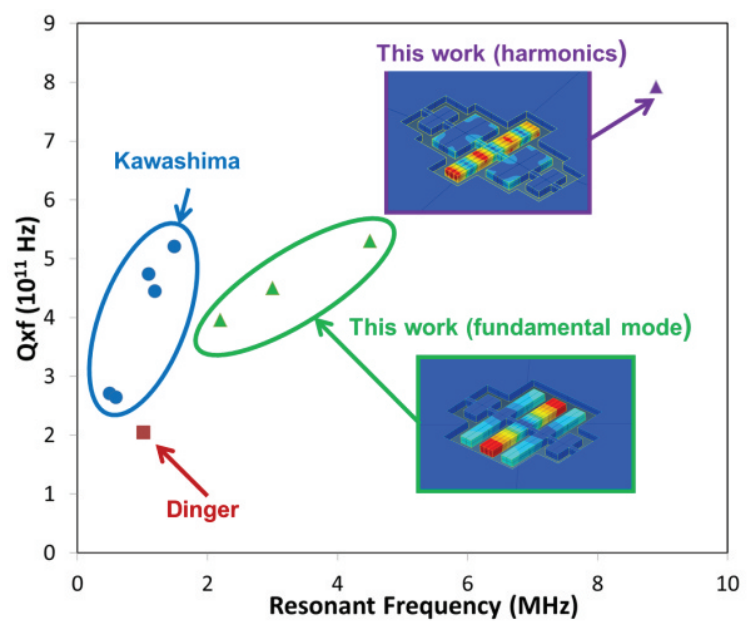

Fig.13 Figure of merit Q.f of 2D-LEM resonators investigated in this work for both fundamental and harmonic modes and 2D-LEM quartz resonators from literature (Dinger1981) (Kawashima1990).

\subsection{Frequency temperature characteristics}

A 2D-LEM type I resonator manufactured with process 2 is mounted on a TO5 header, packaged in a copper case under vacuum. Packaged resonator is put in climatic chamber to perform frequency temperature characteristics, temperature was measured by a platinum sensor close to the packaged resonator. Figure.14 shows the normalized frequency temperature characteristics of a 2D-LEM type I resonator manufactured with process 2 for fundamental mode vibrating at $2.2 \mathrm{MHz}$ for temperatures from -40 to $30^{\circ} \mathrm{C}$. The turn over temperature point of the FEM simulations was $10^{\circ} \mathrm{C}$ whereas the experimental turn over point was $-9.5^{\circ} \mathrm{C}$. However the trend of the curve is the same in experimental and simulated data.

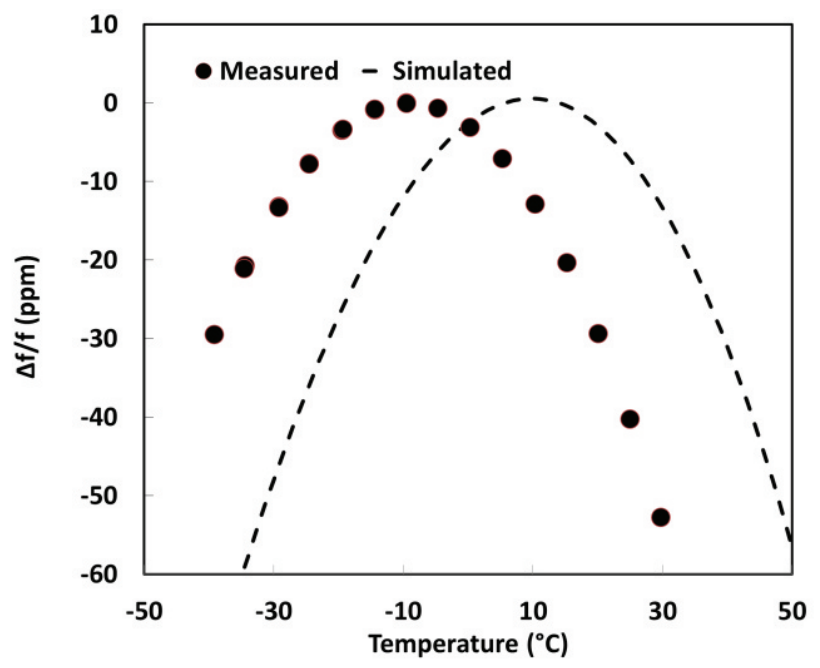

Fig.14 Frequency temperature characteristics of the fundamental mode of type I resonator vibrating at $2.2 \mathrm{MHz}$

A shift is again observed for the first harmonic mode vibrating at $6.6 \mathrm{MHz}$ as shown in Figure 15: the FEM simulations turn over point was $123^{\circ} \mathrm{C}$ whereas experimental turn over point was $99^{\circ} \mathrm{C}$. 


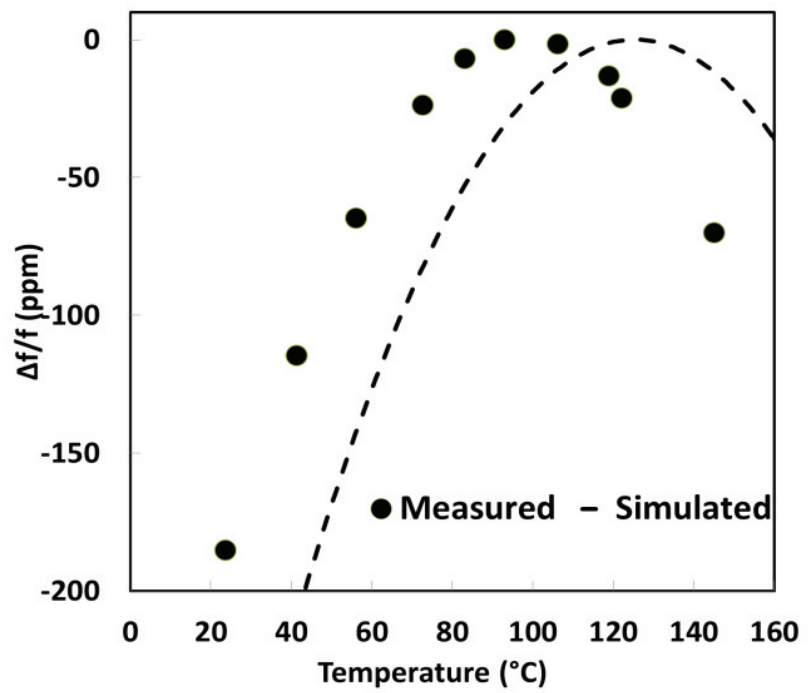

Fig.15 Frequency temperature characteristics of the first harmonic mode of type I resonator vibrating at $6.6 \mathrm{MHz}$ (fundamental mode vibrating at $2.2 \mathrm{MHz}$ ).

The downshifts of the turn over temperature point by almost $20^{\circ} \mathrm{C}$ for the fundamental mode and $23^{\circ} \mathrm{C}$ for the first harmonic are not fully understood yet. An offset of one degree along $\mathrm{X}$ axis in the cut angle can almost cause a $10^{\circ} \mathrm{C}$ variation of the turn over temperature point, as shown in Figure 16 where FEM simulations of normalized frequency temperature characteristics for angle values of $\Theta=-2,0$ and $+2^{\circ}$ aound $\mathrm{X}$-axis along with experimental data, turn over point is $+30^{\circ} \mathrm{C}$ for $\Theta=-2^{\circ}$, and $-15^{\circ} \mathrm{C}$ for $\Theta=$ $+2^{\circ}$ (in the FEM simuations the structure is rotated by an angle $\Theta$ around the fixed axis coordinates which is equivalent to a rotation of in the crystalline axis of $-\Theta)$ but it seems unlikely due to the high angle value $\left(\approx 1.5^{\circ}\right)$ required to fit the data. Rotations around the two other cristalline axes did not lead to significant shift of the turn over point. Another hypothesis implies an effect of the surface roughness that can shift the turn over point as it had been observed in our team for flexural quartz resonators: turn over point was shifted to lower temperatures with increasing surface roughness. Further investigations are necessary to confirm the precise value of the resonators crystalline orientation and to measure frequency temperature characteristics of resonators manufactured with process 1 without the pyramids on the backside cavities

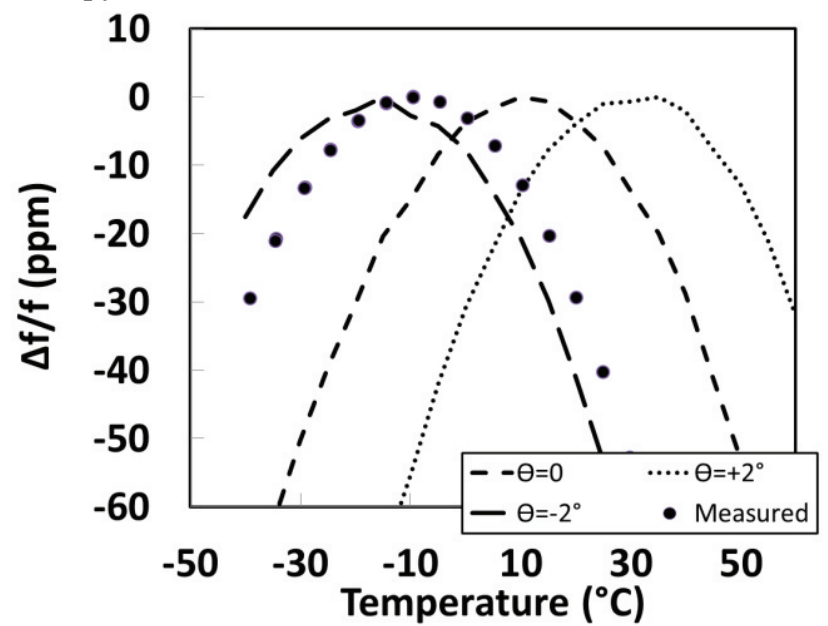

Fig.16 Frequency temperature characteristics of the first harmonic mode of type I resonator vibrating at $2.2 \mathrm{MHz}$ and FEM simulations with differents angles around de $\mathrm{X}$-axis. Indicated values of $\Theta$ correspond to the values entered in the FEM simulation software.

\section{DISCUSSION}

In this section, the discrepancy between the measured and theoretical quality factor are discussed. As mentioned earlier, resonators made with process 2 showed qualitatively lower $Q$ factor than those obtained with process 1 . Moreover, experimental quality factors obtained are lower than those predicted.

\subsection{Process 1 and process 2}

Resonators manufactured with process 1 showed better $Q$ factors than the ones made with process 2 . Figure 17 a) shows the backside of a resonator manufactured with process 1, apart from some micro-masking induced by contaminents, the surface is smooth with $\mathrm{R}_{\mathrm{a}}=4 \mathrm{~nm}$ measured with a mechanical profilometer (KLA Tencor D-100). The reduced quality factors of the process 2 
are likely due to the presence of pyramids on the back side as illustrated in Figure 17b) induced by the wet chemical etching using $\mathrm{HF}-\mathrm{NH}_{4} \mathrm{~F}$. As there are no pyramids on the top face, the structure obtained with process 2 is less symmetrical than in the case of process 1 and is also way more rougher with $\mathrm{R}_{\mathrm{a}}=1.9 \mu \mathrm{m}$, which may amplify the loss energy through the anchors: this lowers $Q_{\text {anchor }}$ hence the overall decrease of the experimental $Q$ factor.

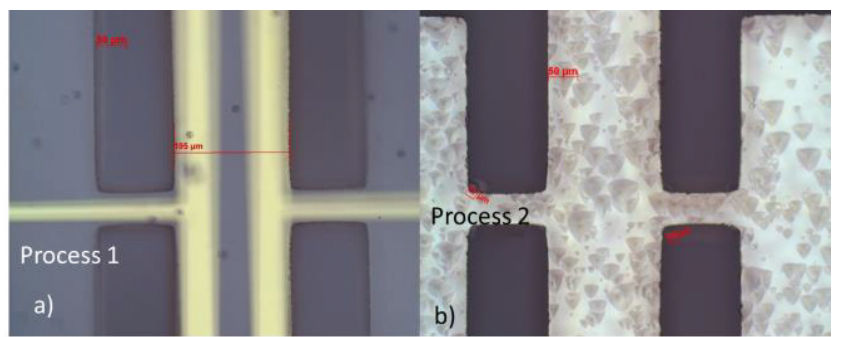

Fig. 17 a) Optical image of the back side of a resonator using the double steps DRIE process (process 1), b) optical image of the back side of a resonator using wet etching (process 2) and presence of pyramids with several tens of microns diameters MF $=200$.

\subsection{Experimental Q factors}

The simulated structure for the calculation of $Q_{\text {anchor }}$ is not exactly the one obtained with process 1 . Indeed, in the simulations the sidewalls were perfectly vertical whereas DRIE cannot ensure a perfect verticality as illustrated in Figure 8 . FEM simulations give the dependency of the $Q_{\text {anchor }}$ versus sidewall angle in Figure.8. It appears that energy trapping in the vibrating part becomes rapidly less efficient with an, even slightly, non-vertical sidewall angle. To optimize $Q_{a n c h o r}$, resonator design must be made so that the mounting areas match vibration nodes where the strain energy is almost null. As soon as the sidewall angle is non-zero, the structure is no longer symmetrical and vibration nodes are slightly displaced whereas the mounting areas are in the same place that for the ideal structures. For an angle around $3^{\circ}$ which is likely to be reached with the current process 1 , as seen in the detail of Figure 18, Q anchor falls down around 400000 for the $3 \mathrm{MHz}$ resonator as shown in Figure.18 and 500000 for the $2.2 \mathrm{MHz}$ and explains the experimental values.

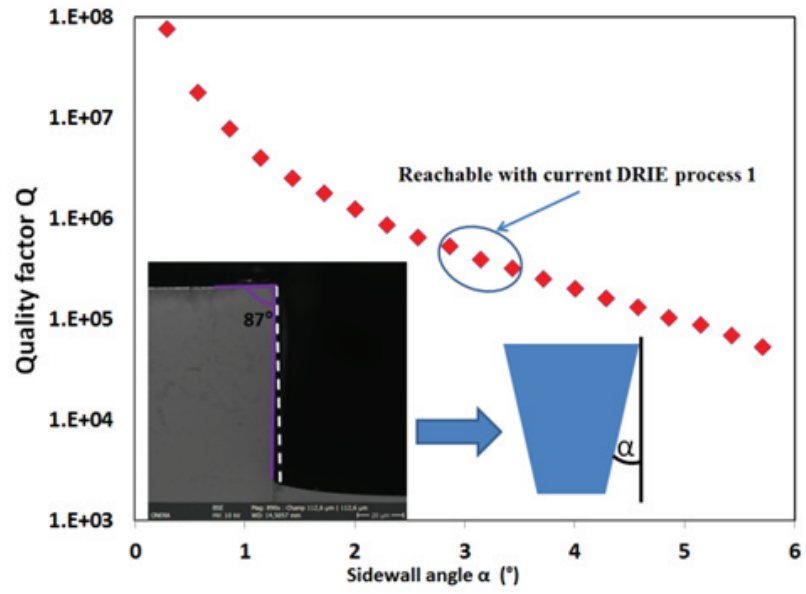

Fig.18 Simulations using FEM of the Q Qanchor vs sidewall angle for a 3 MHz type I resonator. In detail, scanning electron microscopy (SEM) of a $90 \mu \mathrm{m}$ deep cross section beam

One can note that the larger resonators with lower resonance frequencies present better performances than the smaller ones vibrating at higher resonance frequencies - and that was also observed in the work of Kawashima (Kawashima1990). Type II resonators showed better performances than equivalent previous standard type I design, which seems to indicate either a more efficient design with the out-of-phase extension-compression mode of the lateral beams or a less sensitive dependence of $Q_{a n c h o r}$ with sidewall angle.

\section{Conclusions}

In this paper, new 2D-LEM quartz resonators with potential Time\&Frequency applications have been demonstrated with first prototypes showing encouraging electro-mechanical characteristics providing a $Q . f$ product near $10^{12} \mathrm{~Hz}$ and a $Q$ factor at the state of art for DRIE made quartz resonators. The two main limiting damping mechanisms have been identified: the energy losses arise from the viscous dissipation in the gold layer forming the electrodes and the mounting areas. First characterizations of harmonic modes were performed and showed promising results. Future work will be focused on increasing the resonance frequency and maintaining or improving the quality factor thanks to better control of the sidewall angle. 


\section{REFERENCES}

\subsection{Journal article}

Akhiezer A (1939) On the absorption of sound in solids. Journal of Physics (Moscow), vol. 1, no. 1, pp. 277-287.

Pelle R, Hedlund C, Katardjiev I V, Bäcklund Y (1998) Etch rates of crystallographic planes in Z -cut quartz-experiments and simulations, J Micromech. Microeng. 8, 1-6. https://doi.org/10.1088/0960-1317/8/1/001

Ghaffari S, Chandorkar SA., Wang Q, Ng EJ, Ahn CH, Hong V, Yang Y, Kenny TW. (2013) Quantum limit of quality factor in silicon micro and nano mechanical resonators. Sci. Rep $3: 3244$. https://doi.org/10.1038/srep03244

HyunHo D, JungHun K, SeokHuyn L, KiWoong W (1996) Mechanism of selective SiO2/Si etching with fluorocarbon gases (CF4 , C4F8) and hydrogen mixture in electron cyclotron resonance plasma etching system. Journal of Vacuum Science \& Technology A 14, 2827. https://doi.org/10.1116/1.580231

Le Foulgoc B, Bourouina T, Le Traon O, Bosseboeuf A, Marty F, Breluzeau, Grandchamp JP, Masson S (2006) Highly decoupled single-crystal silicon resonators: an approach for the intrinsic quality factor. J. Micromech. Microeng. 16 s45-53. http://doi.org/10.1088/0960-1317/16/6/s08

Le Traon O, Masson S, Chartier C, Janiaud D (2010) LGS and $\mathrm{GaPO}_{4}$ piezoelectric crystals: New results. Solid State Sciences 12 318-324. https://10.1016/j.solidstatesciences.2009.06.032

Sankaran A, Kushner MJ (2004) Integrated feature scale modeling of plasma processing of porous and solid SiO2. I. Fluorocarbon etching. Journal of Vacuum Science \& Technology A: Vacuum, Surfaces, and Films, $22(4)$, p.1242. https://doi.org/10.1116/1.1764821

Kutsuwada, H. et al., (2017). Sensors and Actuators A : Physical Fabrication of a true-Gaussian-shaped quartz crystal resonator. Sensors \& Actuators: A. Physical, 260, pp.58-61. Available at: http://dx.doi.org/10.1016/j.sna.2017.04.019

Abe, T. \& Esashi, M., (2000). One-chip multichannel quartz crystal microbalance (QCM) fabricated by Deep RIE. ,Sensors \& Actuators: A. Physical, 82, pp.139-143. https://doi.org/10.1016/S0924-4247(99)00330-1

Xereas G, Chodavarapu VP (2015) Wafer-Level Vacuum-Encapsulated Lamé Mode Resonator With f-Q Product of $2.23 \times 1013$ Hz. IEEE Electron Device Letters, vol. 36, no. 10, pp1079-1081. 10.1109/LED.2015.2464713

Nguyen CTC,(2007) MEMS Technology for Timing and Frequency Control. IEEE Transactions on Ultrasonics, Ferroelectrics, and Frequency Control vol.54, no. 2.10.1109/TUFFC.2007.240

Van Beek JTM, Puers R (2012) A review of MEMS oscillators for frequency reference and timing applications. Journal of Micromechanics and Mircroengineering, 22, 013001 topical review. 10.1088/0960-1317/22/1/013001

Jokic I, Frantlovix M, Djuric Z, Dukic ML (2015) RF MEMS / NEMS RESONATORS FOR WIRELESS COMMUNICATION SYSTEMS AND ADSORPTION- DESORPTION PHASE NOISE Facta Universitatis, Electronics and Energetics, vol. 28, no. 3, pp 345-381. 10.2298/FUEE1503345J

Kaajakari V, Kosikinen JK, Mattila T (2005) Phase Noise in Capacitively Coupled Micromechanical Oscillators IEEE Transactions on Ultrasonics, Ferroelectrics, and Frequency Control vol.52, no. 12. 10.1109/TUFFC.2005.1563277

Khosla KE, Vanner MR, Ares N, Laird EA (2018) Displacemon Electromechanics : How to Detect Quantum Interference in a Nanomechanical Resonator, Physical Review X, vol. 8, no. 2, pp 1-15. 10.1103/PhysRevX.8.021052

Pirkkalainen JM, Damskagg E, Brandt M, Massel F, Sillanpaa MA (2015) Squeezing of Quantum Noise of Motion in a Micromechanical Resonator, Physical Review Letters, vol. 115, no 243601. 10.1103/PhysRevLett.115.243601 
Rivière R, Deléglise, Weis S, Gavartin E, Arcizet O, Schliesser A, Kippenberg TJ (2011) Optomechanical sideband cooling of a micromechanical oscillator close to the quantum ground state, Physical Review A, vol.83, 063835, pp 1-9. 10.1103/PhysRevA.83.063835

Connell A, Hofheinz M, Ansmann et al. (2010) Quantum ground state and single-phonon control of a mechanical resonator, Nature, vol. 464, no. 7289, pp 697-703. 10.1038/nature08967

\subsection{Proceeding-Article by DOI}

Bourgeteau B, Lévy R, Janiaud D, Lavenus P, Le Traon O (2015) Quartz resonator for MEMS oscillator. 2014 European Frequency and Time Forum, EFTF 2014, pp.286-289. https://doi.org/10.1109/EFTF.2014.7331488

Bourgeteau-Verlhac B, Lévy R, Perrier T, Lavenus P, Guérard J, Le Traon O (2016) Gold Thin Film Viscoelastic Losses of a Length Extension Mode Resonator. Eur. Freq. Time Forum, pp. 1-4. https://doi.org/10.1109/EFTF.2016.7477774

Boy JJ, Tavernier H, Vacheret X, Laroche T, Clairet A (2011) Collective fabrication of $20 \mathrm{MHz}$ resonators by deep Reactive Ion Etching on 3" quartz wafers. Proceedings of the IEEE International Frequency Control Symposium and Exposition, pp.3-7. https://doi.org/10.1109/FCS.2011.5977812

Dinger R.J. (1981) A minature quartz resonator vibrating at 1 MHz. Proc. 35 th Ann. Freq Control Symposium, pp.144-148. https://doi.org/10.1109/FREQ.1981.200468

Kawashima H, Nakazato M (1990) Variational analysis of new shape length extensional mode quartz crystal resonator taking account of lateral motion Proc. 35 th Ann. Symp. Freq. Control, pp. 378-386. https://doi.org/10.1109/FREQ.1990.177523

Le Traon O, Bahriz M, Ducloux O, Masson S, Janiaud D (2011) A Micro-Resonator for Fundamental Physics Experiments and its Possible Interest for Time and Frequency Applications. Joint Conference of the IEEE International Frequency Control and the European Frequency and Time Forum (FCS) Proceedings. https://doi.org/10.1109/FCS.2011.5977866

Grousset, S. et al., (2014). Quartz-based vibrating MEMS fabricated using a wafer-bonding process with sealed cavities. , pp.5-8. 10.1109/FCS.2014.6859861

Kubena, R.L. et al.,(2017). A Fully Integrated Quartz MEMS VHF TCXO. IEEE Transactions on Ultrasonics, Ferroelectrics, and Frequency Control, 3010(c), pp.3-7. 10.1109/TUFFC.2017.2786248

Kamijo, A. et al., (2014). Wafer-level quartz dry etching technology. IFCS 2014 - 2014 IEEE International Frequency Control Symposium, Proceedings, 1035, pp.6-9. 10.1109/FCS.2014.6859862

Vig JR, (2016) Quartz Crystal Resonators and Oscillators for Frequency Control and Timing Applications-A Tutorial, July 2016 Rev. 8.5.7.0. 10.13140/2.1.2134.0962

\subsection{Book}

Brand O, Dufour I, Heinrich SM, Josse F (2015) Resonant MEMS-Fundamentals, Implementation and Application, First Edition. 2015 Wiley-VCH Verlag \& Co. KGaA, Boschstr. 12, 69469 Weinheim, Germany, pp 55-71

\subsection{Patent}

O. Le Traon, (2013) "Structure plane de résonateur mécanique découplé par des vibrations de flexion et d'extension compression," n 13/00322. 